\title{
Optimal Hold Functions for Sampled Data Regulation*
}

\author{
Y.-C. JUAN $\dagger$ and P. T. KABAMBA $\dagger \ddagger$
}

Key Words-Digital control; feedback control; optimal regulators; sampled data systems.

\begin{abstract}
This paper investigates the use of Generalized Sampled Data Hold Function Control (GSHF) to optimize quadratic measures of performance in sampled data control loops. The idea of GSHF control is to use sampled data feedback, but consider the hold function as a design parameter. Explicit solutions are given for the GSHF versions of the optimal $L Q$ and LQG regulators. The questions of existence, uniqueness and computation of optimal hold functions are treated. An example is presented.
\end{abstract}

\section{Introduction}

THE TRADITIONAL approach to sampled data control assumes that the discrete time plant model is obtained from a continuous time system by using prespecified hold devicestypically, zero-order or first-order hold (Franklin and Powell, 1980). A digital computer then implements a discrete time control law. In such a scheme, the subsequent intersampling behavior of the controlled continuous time system is fairly well understood (De Souza and Goodwin, 1984; Berger, 1985; Urikura and Nagata, 1987; Sirisena, 1985; Franklin and Emami-Naeini, 1986). Recently, however, a new approach to sampled data control has been introduced (Chammas and Leondes, 1978a, b, 1979). This method is called "Generalized Sampled-Data Hold Function Control" (GSHF), and its original feature is to consider the hold function as a design parameter. Until now, studies on GSHF control have been primarily focused on the resulting discrete time system, and little has been said about the intersampling behavior of the controlled continuous time system (Juan and Kabamba, 1987; Kabamba, 1986, 1987; Kaczorek, 1985, 1986; Zavgren, 1983; Zeng, 1985; Zavgren and Tarn, 1984; Tarn et al., 1988; Greshak and Verghese, 1982).

In this brief paper, we present solutions of optimal design problems for GSHF control where the performance index penalizes the intersampling behavior of the closed loop system. This is accomplished by using penalty indices which are time integrals of a function of the state and control of the regulated continuous time system. The optimization problem then becomes a standard optimal control problem, but where the "control variable" is the hold function itself. Existence and uniqueness of an optimal hold function is proved for a wide class of optimal GSHF regulation problems. The specific problems solved in this paper are GHSF-control versions of the linear-quadratic and linear-quadratic Gaussian regulators. The solution of these problems, together with our results on existence and uniqueness of optimal hold functions constitute the original contribution of this study.

* Received 31 May 1988; revised 7 March 1990; received in final form 23 July 1990 . The original version of this paper was not presented at any IFAC meeting. This paper was recommended for publication in revised form by Associate Editor R. V. Patel under the direction of Editor $H$. Kwakernaak. Partially supported by NSF under Grant ECS-8722244.

+ Aerospace Engineering Department, The University of Michigan, Ann Arbor, MI 48109-2140, U.S.A.

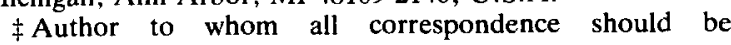
addressed.
Several authors have investigated the intersampling behavior of sampled data control systems. Dorato and Levis (1971) showed how to compute the penalty matrices of a discretized LQG problem based on those of a continuous time LQG problem, assuming zero-order hold. De Souza and Goodwin (1984) presented a method for computing the time-varying output covariance between sampling instants. Berger (1985) suggested that the output between samples can be predicted by a linear function and used this in adaptive control. Ripple-free deadbeat control for sampled data systems was investigated in Urikura and Nagata (1987) and Sirisena (1985), and a necessary and sufficient condition was given. Franklin and Emami-Naeini (1986) showed that a continuous time internal model is necessary and sufficient to provide ripple-free response for the robust servomechanism. However, all these studies assume that the hold functions are not part of the design problem.

Generalized sampled data hold function control has been investigated for finite dimensional, continuous time systems (Kabamba, 1987; Juan and Kabamba, 1987; Chammas and Leondes, 1978a, b; Kabamba, 1986), finite dimensional discrete time systems (Kaczorek, 1985, 1986), and infinite dimensional, continuous time systems (Zavgren, 1983; Zeng, 1985; Zavgren and Tarn, 1984; Tarn et al., 1988). The kindred idea of periodic compensation of time invariant systems has also received attention (Greshak and Verghese, 1982; Khargonekar et al., 1985). The primary concern of these studies has been the properties of the discrete time closed loop system, because they determine important characteristics of the continuous time system such as stability and deadbeat response. As a consequence, the intersampling behavior of continuous time systems under GSHF regulation has received little attention. However, in Kabamba (1987) it was shown that in GSHF control, this intersampling behavior may be unsatisfactory. This phenomenon motivates the present study.

\section{Problem formulation, notations and definitions}

We use the following standard notations: superscript $T$ denotes matrix transpose; $E(\cdot)$ denotes expected value; $\operatorname{tr}(\cdot)$ denotes the trace of a matrix; $\delta(\cdot)$ denotes the Kronecker symbol in both the discrete time and continuous time case; $I_{D}$ denotes the identity matrix of order $p$. Let $X \in \mid R^{m \times n}$, and denote the columns of $X$ as $x_{i}, i=1, \ldots, n$, i.e. $X=$ $\left[x_{1}, \ldots, x_{n}\right]$. The vec operator on $X$ is defined as $\operatorname{vec}(X)=\operatorname{col}\left[x_{1}, x_{2}, \ldots, x_{n}\right]$, where $\operatorname{vec}(X) \in \mid R^{m n \times 1}$.

We consider finite dimensional linear time invariant, continuous time systems under sampled-data regulation as follows (see Fig. 1):

Plant and sampler:

$$
\begin{gathered}
\dot{x}(t)=A x(t)+B u(t)+w(t) \\
\zeta(k)=C x(k T)+v(k) .
\end{gathered}
$$

Digital compensator

$$
\begin{gathered}
\xi(k+1)=P \xi(k)+U \xi(k) \\
\gamma(k)=S \xi(k)+V \xi(k) .
\end{gathered}
$$




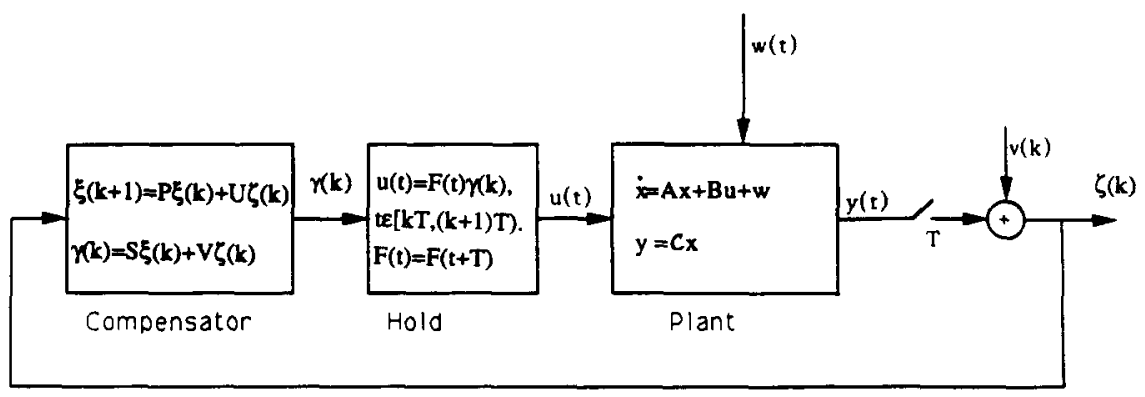

FIG. 1. Feedback configuration.

Digital-to-analog conversion (Hold device)

$$
\begin{gathered}
u(t)=F(t) \gamma(k), \quad t \in[k T,(k+1) T) \\
F(t)=F(t+T), \forall t
\end{gathered}
$$

where $x(t) \in \mid R^{n}$ is the plant state vector; $u(t) \in \mid R^{m}$ is the control input; $w(t) \in \mid R^{p}$ is a disturbance vector, $\zeta(k) \in \mid R^{p}$ and $v(k) \in \mid R^{p}$ are the discrete measurement vector and discrete measurement noise vector, respectively; $\xi(k) \in \mid R^{q}$ is the compensator state vector; $\gamma(k) \in \mid R^{r}$ is the compensator output; $T>0$ is the sampling period; $F(t) \in \mid R^{r \times q}$ is a $T$-periodic integrable and bounded matrix representing a hold function; and the real matrices $A, B, C, P, U, S, V$ have appropriate dimensions. Without loss of generality, we assume that the matrices $C$ and $(S, V)$ have linearly independent rows.

The formalism of equations (2.1)-(2.6) is quite general. Zero-order hold (first-order hold, $i$ th-order hold) control is obtained by letting the hold function $F(t)$ be a constant (first-degree polynomial, $i$ th-degree polynomial, respectively). Also, by letting $q=0, r=p$, and $V=I_{p}$, we obtain the formalism of GSHF control with direct output feedback used e.g. in Kabamba (1987).

For a given hold function $F(t), t \in[0, T)$, the problem of designing the matrices $P, U, S, V$ for performance of the corresponding discrete time system has been extensively treated in the literature [see e.g. Franklin and Powell (1980)]. Our objective in this paper is, for a given compensator $(2.3)-(2.4)$, to determine time histories of the hold function $F(t)$ in $(2.5)$, (2.6) that will optimize various performance criteria associated with the sampled data system (2.1)-(2.6)

Upon loop closure, the state and control between samples satisfy

$$
\begin{gathered}
x(k T+t)=\Phi(t) x(k T)+D(t) S \xi(k)+D(t) V v(k) \\
+\omega(k T+t), \quad t \in[0, T] \\
u(k T+t)= \\
F(t) V C \times(k T)+F(t) S \xi(k) \\
+F(t) V v(k), \quad t \in[0, T]
\end{gathered}
$$

where

$$
\Phi(t)=\exp (A t)+D(t) C, \quad t \in[0, T]
$$$$
D(t)=\int_{0}^{t} \exp (A(t-\tau)) B F(\tau) \mathrm{d} \tau, \quad t \in[0, T]
$$

$\omega(k T+t)=\int_{k T}^{k T+t} \exp (A(k T+t-\tau)) w(\tau) \mathrm{d} \tau$,

$$
t \in[0, T] .
$$

Defining

$$
\begin{gathered}
x_{a}(k)=\left[x^{\mathrm{T}}(k T), \xi^{\mathrm{T}}(k)\right]^{\mathrm{T}} \in \mid R^{n+q} \\
\Psi_{\mathrm{a}}=\left[\begin{array}{cc}
\exp (A T)+D(T) V C & D(T) S \\
U C & P
\end{array}\right] \in \mid R^{(n+q) \times(n+q)} \\
D_{a}=\left[\begin{array}{c}
D(T) V \\
U
\end{array}\right] \in \mid R^{(n+q) \times p}
\end{gathered}
$$

$$
\omega_{a}(k)=\left[\begin{array}{c}
\omega(k) \\
0
\end{array}\right] \epsilon \mid R^{n+q}
$$

then the closed loop equations for the discrete time system are

$$
x_{a}(k+1)=\Psi_{a} x_{a}(k)+D_{a} v(k)+\omega_{a}(k) .
$$

The closed loop monodromy matrix is defined as $\Psi_{a}$ in (2.13), (2.16) and denotes the state transition matrix of the regulated discrete-time system over one period.

Definition. The design problem of finding an optimal hold function $F(\tau), \tau \in[0, T]$ is called a fixed monodromy (free monodromy) problem if $D(T)$ in (2.10) is specified (not specified).

A fixed monodromy problem must therefore satisfy a design constraint of the form

$$
D(T)=G,
$$

where typically, the matrix $G$ is chosen such that the closed loop monodromy matrix $\Psi_{a}$ of (2.13) defines a stable discrete time system (2.16)

\section{Linear-quadratic Gaussian regulation}

Throughout this section we assume that $w(t)$ and $v(k)$ of (2.1)-(2.3) are stationary Gaussian processes satisfying

$$
\begin{gathered}
E[w(t)]=0, \quad t \in \mid R ; \\
E[v(k)]=0, \quad k \in \mid N ; \\
E\left[w(t) v^{\mathrm{T}}(k)\right]=0, \quad t \in|R, \quad k \in| N ; \\
E\left[w(t) w^{\mathrm{T}}(\tau)\right]=R_{w} \delta(t-\tau), t, \quad \tau \in \mid R, \quad R_{w} \geq 0 ; \\
E\left[v(k) v^{\mathrm{T}}(\iota)\right]=R_{v} \delta(k-\imath), k, \quad \iota \in \mid N, \quad R_{v} \geq 0 ; \\
E\left[w(t) x^{\mathrm{T}}(0)\right]=0, \quad t \in \mid R ; \\
E\left[v(k) x^{\mathrm{T}}(0)\right]=0, \quad k \in \mid N .
\end{gathered}
$$

Equation (2.9) implies that $\omega(k)$ is a stationary zero-mean white Gaussian sequence with covariance kernel

$$
\begin{gathered}
E\left[\omega(k) \omega^{\mathrm{T}}(\iota)\right]=R_{\omega}(T) \delta(k-\iota), \\
R_{\omega}(T)=\int_{0}^{T} \exp (A(T-\tau)) R_{w} \exp \left(A^{\mathrm{T}}(T-\tau)\right) \mathrm{d} \tau
\end{gathered}
$$

The performance index has the form

$$
J=\lim _{t \rightarrow \infty} E\left\{\frac{1}{t_{f}} \int_{0}^{t_{f}}\left(x^{\mathrm{T}} Q x+u^{\mathrm{T}} R u\right) \mathrm{d} t\right\},
$$

where $Q \in\left|R^{n \times n}, Q=Q^{\mathrm{T}}, \quad Q \geq 0, \quad R \in\right| R^{m \times m}, \quad R=R^{\mathrm{T}}$, $R>0$. The criterion (3.10) can also be computed as follows (see Juan, 1988).

Proposition 1. Suppose a hold function $F(t), t \in[0, T]$ stabilizes asymptotically $(2.16)$. Then the criterion $(3.10)$ has the form

$$
J=\lim _{k \rightarrow \infty} E\left\{\frac{1}{T} \int_{k T}^{(k+1) T}\left(x^{\mathrm{T}} Q x+u^{\mathrm{T}} R u\right) \mathrm{d} t\right\} .
$$


Proposition 2. Suppose a hold function $F(t), \quad t \in[0, T]$ stabilizes (2.16) asymptotically. Then the criterion (3.10) can be computed as follows

$$
J=\frac{1}{T} \operatorname{tr}\left\{L_{a} M_{a}(T)+R_{v} N_{a}(T)+Q P_{a}(T)\right\}
$$

where $L_{a} \in\left|R^{(n+q) \times(n+q)}, \quad N_{a}(t) \in\right| R^{p \times p}, P_{a}(t) \in \mid R^{n \times n}$ are obtained as follows

$$
\begin{aligned}
& \dot{D}(t)=A D(t)+B F(t), \quad D(0)=0 \\
& \dot{M}_{a}(t)=\left[\begin{array}{c}
\exp \left(A^{\mathrm{T}} t\right)+C^{\mathrm{T}} V^{\mathrm{T}} D^{\mathrm{T}}(t) \\
S^{\mathrm{T}} D^{\mathrm{T}}(t)
\end{array}\right] \\
& \times Q[\exp (A t)+D(t) V C, D(t) S] \\
& +\left[\begin{array}{c}
C^{\mathrm{T}} V^{\mathrm{T}} F^{\mathrm{T}}(t) \\
S^{\mathrm{T}} F^{\mathrm{T}}(t)
\end{array}\right] R[F(t) V C, F(t) S] ; \quad M_{a}(0)=0 \\
& {\left[\begin{array}{cc}
\exp (A T)+D(T) V C & D(T) S \\
U C & P
\end{array}\right]} \\
& \times L_{a}\left[\begin{array}{cc}
\exp \left(A^{\mathrm{T}} T\right)+C^{\mathrm{T}} V^{\mathrm{T}} D^{\mathrm{T}}(T) & C^{\mathrm{T}} U^{\mathrm{T}} \\
S^{\mathrm{T}} D^{\mathrm{T}}(T) & P^{\mathrm{T}}
\end{array}\right] \\
& +\left[\begin{array}{c}
D(T) V \\
U
\end{array}\right] R_{v}\left[V^{\mathrm{T}} D^{\mathrm{T}}(T), U^{\mathrm{T}}\right]+\left[\begin{array}{cc}
R_{\omega}^{(\mathrm{T})} & 0 \\
0 & 0
\end{array}\right]-L_{a}=0 . \\
& N_{a}(t)=\int_{0}^{t}\left[N^{\mathrm{T}} D_{(\mathrm{r})}^{\mathrm{T})} Q D(\tau) V+V^{\mathrm{T}} F^{\mathrm{T}}(\tau) R F(\tau) V\right] \mathrm{d} \tau \\
& P_{a}(t)=\int_{0}^{t} R_{\omega}(\tau) \mathrm{d} \tau .
\end{aligned}
$$

Proof. First rewrite (2.10) as (3.13). Define $L_{a}=$ $\lim _{k \rightarrow \infty} E\left[x_{a}(k) x_{a}^{\top}(k)\right]$. The stability of (2.16) implies that $L_{a}$ can be computed by (3.15). Moreover, simple algebraic manipulations based on (2.7)-(2.11) imply that the cost (3.11) can be computed as (3.12) subject to (3.13)-(3.17).

(a) Free monodromy.

Proposition 3. A hold function $F(t), t \in[0, T]$ which stabilizes (2.16) asymptotically and minimizes (3.10) must satisfy

$$
\begin{aligned}
B^{\mathrm{T}} \Psi(t) & -2 R F(t)[V C, S] L_{a}\left[\begin{array}{ll}
C^{\mathrm{T}} & V^{\mathrm{T}} \\
& S^{\mathrm{T}}
\end{array}\right] \\
& -2 R F(t) V R_{v} V^{\mathrm{T}}=0
\end{aligned}
$$

where $\Psi(t) \in \mid R^{n \times r}$ satisfies

$$
\begin{gathered}
\dot{\Psi}(t)=-A^{\mathrm{T}} \Psi(t)+2 Q[\exp (A t)+D(t) V C, D(t) S] L_{a} \\
\times\left[\begin{array}{cc}
C^{\mathrm{T}} & V^{\mathrm{T}} \\
S^{\mathrm{T}}
\end{array}\right]+2 Q D(t) V R_{v} V^{\mathrm{T}} \\
\Psi(T)=-2\left[I_{n}, 0\right] K_{a}\left\{\left[\begin{array}{cc}
\exp (A T)+D(T) V C & D(T) S \\
U C & P
\end{array}\right]\right. \\
\left.\times L_{a}\left[\begin{array}{cc}
C^{\mathrm{T}} & V^{\mathrm{T}} \\
S^{\mathrm{T}}
\end{array}\right]+\left[\begin{array}{cc}
D(T) & V \\
U
\end{array}\right] R_{v} V^{\mathrm{T}}\right\} \\
{\left[\begin{array}{cc}
\exp \left(A^{\mathrm{T}} T\right)+C^{\mathrm{T}} V^{\mathrm{T}} D^{\mathrm{T}}(T) & C^{\mathrm{T}} U^{\mathrm{T}} \\
S^{\mathrm{T}} D^{\mathrm{T}}(T) & P^{\mathrm{T}}
\end{array}\right]} \\
\times K_{a}\left[\begin{array}{cc}
\exp (A T)+D(T) V C & D(T) S \\
U C & P
\end{array}\right] \\
+M_{a}(T)-K_{a}=0
\end{gathered}
$$

and the matrices $D(t), L_{a}$ and $M_{a}(t)$ are given by (3.13)-(3.15).

Proof. The optimization of (3.12) subject to (3.13)-(3.17) is performed using standard optimal control theory, but where the state variable is the matrix $D(t)$ and the control input is the hold function $F(t)$, yielding (3.18)-(3.21). See Juan (1988) for details.
Equations (3.13)-(3.21) define a two point boundary value problem for the two $n \times r$ matrices $D(t)$ and $\Psi(t)$. The first-order gradient algorithm of Bryson and Ho (1975) has been used successfully to compute solutions to these equations. The examples we have treated suggest that in general, (3.13)-(3.21) always have a solution when the triple $(A, B, C)$ of $(2.1)-(2.2)$ is minimal, but that this solution is not in general unique. However, in the case of fixed monodromy, we can guarantee both existence and uniqueness.

(b) Fixed monodromy.

Proposition 4. If the triple $(A, B, C)$ of $(2.1)-(2.2)$ is minimal, and the matrix $G$ of $(2.17)$ is such that the system (2.16) is asymptotically stable, then for almost all $T$, the hold function $F(t), t \in[0, T]$ which minimizes (3.10) subject to (2.1)-(2.6), (3.1)-(3.9), (2.17) exists and is unique. It satisfies

$$
\begin{aligned}
& \dot{D}(t)=A D(t)+\frac{1}{2} B R^{-1} B^{\mathrm{T}} \Psi(t) \\
& \times\left\{[V C, S] L_{a}\left[\begin{array}{cc}
C^{\mathrm{T}} & V^{\mathrm{T}} \\
S^{\mathrm{T}}
\end{array}\right]+V R_{v} V^{\mathrm{T}}\right\}^{-1}, \\
& D(0)=0, \quad D(T)=G \\
& \dot{\Psi}(t)=-A^{\mathrm{T}} \Psi(t)+2 Q[\exp (A t)+D(t) V C, D(t) S] L_{a} \\
& \times\left[\begin{array}{c}
C^{\mathrm{T}} V^{\mathrm{T}} \\
S^{\mathrm{T}}
\end{array}\right]+2 Q D(t) V R_{v} V^{\mathrm{T}} \\
& F(t)=\frac{1}{2} R^{-1} B^{\mathrm{T}} \Psi(t)\left\{[V C, S] L_{a}\left[\begin{array}{cc}
C^{\mathrm{T}} & V^{\mathrm{T}} \\
S^{\mathrm{T}}
\end{array}\right]+V R_{v} V^{\mathrm{T}}\right\}^{-1}
\end{aligned}
$$

where $L_{a}$ is the positive semidefinite solution of (3.15).

Proof. See Juan (1988).

Notice that (3.22), (3.23) can be rewritten as a standard Hamiltonian two point bounary value problem for the vectors $\operatorname{vec}(D)$ and $\operatorname{vec}(\Psi)$. The direct solution of (3.22), (3.23) therefore requires only solving linear equations (Bryson and Ho, 1975).

\section{Linear quadratic regulation}

Throughout this section we assume there is no disturbance and no measurement noise: we are regulating the transient behavior of the closed loop system against nonzero initial conditions. In (2.1)-(2.3) we assume:

$$
\begin{gathered}
w(t)=0, \quad v(k)=0, \\
E(x(0))=0, \quad E(\xi(0))=0, \\
E\left(\left[\begin{array}{l}
x(0) \\
\xi(0)
\end{array}\right]\left[x^{\mathrm{T}}(0), \xi^{\mathrm{T}}(0)\right]\right)=X_{0}^{a}
\end{gathered}
$$

and the performance index has the form

$$
J=E\left(\int_{0}^{\infty}\left(x^{\mathrm{T}} Q x+u^{\mathrm{T}} R u\right) \mathrm{d} t\right)
$$

where $Q \in\left|R^{n \times n}, \quad Q=Q^{\mathrm{T}}, \quad Q \geq 0, \quad R \in\right| R^{m \times m}, \quad R=R^{\mathrm{T}}$, $R>0$.

(a) Free monodromy

Proposition 5. A hold function $F(t), t \in[0, T]$ which stabilizes asymptotically $(2.16)$ and minimizes $(4.4)$ subject to (2.1)-(2.6), (4.1)-(4.3) must satisfy (3.13)-(3.21) where

$$
\left[\begin{array}{cc}
R_{\omega}(T) & 0 \\
0 & 0
\end{array}\right]
$$

is replaced by $X_{0}^{a}$ and $R_{v}$ is replaced by 0 . The optimal value of (4.4) is then

$$
J=\operatorname{tr}\left(K_{a} X_{0}^{a}\right)
$$

(b) Fixed monodromy.

Proposition 6. If the triple $(A, B, C)$ of $(2.1)-(2.2)$ is minimal and the matrix $G$ of $(2.17)$ is such that the system 
(2.16) is asymptotically stable then for almost all $T$, the hold function $F(t), t \in[0, T]$ which minimizes (4.4) subject to (2.1)-(2.6), (4.1)-(4.3), (2.17) exists and is unique. It satisfies $(3.22)-(3.24)$ where

$$
\left[\begin{array}{cc}
R_{\omega}(T) & 0 \\
0 & 0
\end{array}\right]
$$

is replaced by $X_{0}^{a}$ and $R_{v}$ by 0 . The optimal value of (4.4) is then given by (4.5).

Remark 1. Propositions 5 and 6 reveal that the linear quadratic regulator is a particular case of linear quadratic Gaussian regulator where $R_{v}=0$ and $X_{0}^{a}$ replaces

$$
\left[\begin{array}{cc}
R_{\omega}(T) & 0 \\
0 & 0
\end{array}\right]
$$

In other words, optimizing the transient performance (4.4) of a noiseless undisturbed system is equivalent to optimizing the steady state performance (3.11) of the same noiseless system under some properly defined perturbations.

Remark 2. Propositions 3, 4, 5 and 6 illustrate the fact that fixed monodromy problems are easier to solve than free monodromy problems. Not only can we guarantee existence and uniqueness of an optimal fixed monodromy hold function, but it can be computed directly at the expense of solving linear equations. On the other hand, for free monodromy problems we cannot guarantee existence of a solution, neither can we guarantee its uniqueness, nor can we guarantee that the iterative algorithm we used will always converge. The hierarchy of difficulty between free and fixed monodromy problems is reminiscent of the problem of optimal $L^{2}$ model reduction (Wilson, 1970) where, if the poles of the optimal reduced order model are free, it is not guaranteed to exist, nor to be unique, nor to be computable by a convergent algorithm; whereas if these poles are fixed, the reduced order model is computed directly by solving linear equations.

\section{Example}

In this section we illustrate the use of the results of Sections 3-4. We compare two sampled data regulators for a given plant with respect to an integral quadratic performance index. The first regulator is obtained by using a standard zero-order hold, preceded by a discrete compensator based on pole assignment. The second regulator is obtained by using the same discrete compensator, but optimizing the hold function with respect to the performance index, under the "fixed monodromy constraint" that the discretized plant in the second configuration be the same as the discretized plant in the first configuration. In other words, we show that it is possible to improve the performance of a feedback loop by adjusting the hold function, without changing the discrete compensator nor the discrete model of the plant. Such an improvement is then due exclusively to a better intersam- pling behavior. Note that the discrete compensator has not been chosen for optimality with respect to the performance index, because this optimization would depend on the hold function, and the effect of optimizing the hold function alone would then be difficult to assesss.

The plant is a simple harmonic oscillator with position measurement of the form:

$$
\begin{aligned}
& \dot{x}=\left[\begin{array}{rr}
0 & -5 \\
5 & 0
\end{array}\right] x+\left[\begin{array}{l}
0 \\
1
\end{array}\right] u \\
& y=\left[\begin{array}{ll}
1 & 0
\end{array}\right] x .
\end{aligned}
$$

The sampling period is $T=1$.

(A) The first controller is implemented using a dynamic compensator and a zero-order hold

$$
u(t)=\gamma(k), \quad t \in[k T,(k+1) T)
$$

The discretized model of the plant is then

$$
\begin{aligned}
x(k+1) & =\left[\begin{array}{rr}
0.2837 & 0.9589 \\
-0.9589 & 0.2837
\end{array}\right] x(k)+\left[\begin{array}{l}
-0.1433 \\
-0.1918
\end{array}\right] \gamma(k) \\
\zeta(k) & =\left[\begin{array}{ll}
1 & 0
\end{array}\right] x(k) .
\end{aligned}
$$

The dynamic compensator is

$$
\begin{aligned}
\xi(k+1) & =\left[\begin{array}{cc}
-0.06732 & 0.3735 \\
0.2057 & -0.5
\end{array}\right] \xi(k)+\left[\begin{array}{c}
0.5673 \\
-0.875
\end{array}\right] \zeta(k) \\
\gamma(k) & =\left[\begin{array}{ll}
-1.5094 & 4.086
\end{array}\right] \xi(k)
\end{aligned}
$$

and has been chosen so that the closed loop eigenvalues are located at $\pm 1.0 \times 10^{-3}$, and $\pm j \times 10^{-3}$. The cost function has the form (4.4), (4.3) where

$$
\begin{aligned}
Q & =\left[\begin{array}{ll}
1 & 0 \\
0 & 1
\end{array}\right] \\
R & =1 \\
X_{0}^{a} & =\left[\begin{array}{llll}
1 & 0 & 0 & 0 \\
0 & 1 & 0 & 0 \\
0 & 0 & 0 & 0 \\
0 & 0 & 0 & 0
\end{array}\right] .
\end{aligned}
$$

For this regulator, the performance index is computed using Proposition 2 which yields

$$
J_{1}=47.82 \text {. }
$$

(B) The second controller consists of a sampler with generalized hold function, together with the same discrete compensator (5.4). The hold function is constrained such that the discretized plant is the same as (5.3). In other words, the closed loop systems $A$ and $B$ have the same dynamics at sampling instants. However, in case $B$, the hold function is chosen so as to optimize the cost function (4.4), (4.3), (5.5), Aplying Proposition 6 yields the hold function of Fig. 2, with

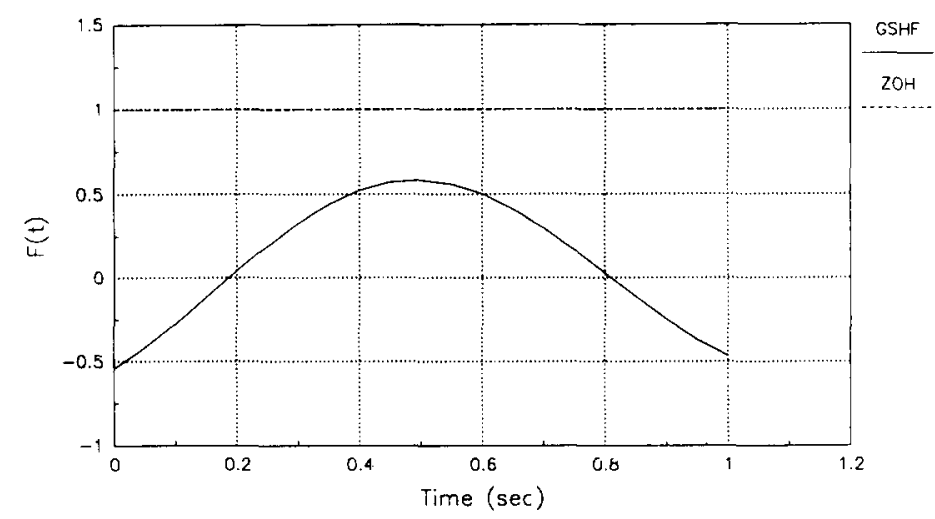

FIG. 2. Generalized sampled data hold functions. 
cost function

$$
J_{2}=25.43
$$

which represents a $47 \%$ improvement over (5.6)

\section{Conclusions}

The main contribution of this paper has been to present methods for optimizing the intersampling behavior of sampled data control systems. The basic premise is that the hold function itself is the design variable. We have presented explicit solutions to the optimal LQ and LQG regulation problems. It is found that for fixed monodromy problems, we can not only guarantee existence and uniqueness of an optimal hold function, but we can compute it directly by solving a linear two point boundary value problem of Hamiltonian structure. This brief paper has only presented the basic theoretical results on optimal sampled data regulation by GSHF. Engineering design issues and detailed examples are considered in Yang and Kabamba (1988) where it is also shown that GSHF control can be used to achieve simultaneous quadratic performance in several control loops using the same controller.

\section{References}

Berger, C. S. (1985). The use of intersampling in adaptive control. IEEE Trans. Aut. Control, AC-30.

Bryson, A. E. and Y.-C. Ho (1975). Applied Optimal Control. Hemisphere, Washington.

Chammas, A. B. and C. T. Leondes (1978a). On the design of linear time invariant systems by periodic output feedback, Parts I and II. Int. J. Control, 27, 885-903.

Chammas, A. B. and C. T. Leondes (1978b). Optimal control of stochastic linear systems by discrete output feedback. IEEE Trans. Aut. Control, AC-23, 921-926.

Chammas, A. B. and C. T. Leondes (1979). On the finite time control of linear systems by piecewise constant output feedback. Int. J. Control, 30, 227-234.

De Souza, C. E. and G. C. Goodwin (1984). Intersample variances in discrete minimum variance control. IEEE Trans. Aut. Control, AC-29.

Dorato, P. and A. H. Levis (1971). Optimal linear regulators: The discrete-time case. IEEE Trans. Aut. Control, AC-16, 613-620.

Franklin, G. F. and J. D. Powell (1980). Digital Control of Dynamic Systems. Addison-Wesley, Reading, MA.

Franklin, G. F. and A. Emami-Naeini (1986). Design of ripple-free multivariable robust servomechanisms. IEEE Trans. Aut. Control, AC-31.
Greshak, J. P. and G. C. Verghese (1982). Periodically varying compensation of time-invariant systems. Syst. Control Lett., 2, 88-93.

Juan, Y.-C. and P. T. Kabamba (1987). Constrained structure control of periodic systems using generalized sampled-data hold functions. Proc. 1987 ACC, pp. 1695-1700, Minneapolis, MN.

Juan, Y.-C. (1988). Control of linear systems with generalized hold functions. Ph.D. Dissertation, The University of Michigan, MI.

Kabamba, P. T. (1986). Monodromy eigenvalue assignment in linear periodic systems. IEEE Trans. Aut. Control, AC-31, 950-952.

Kabamba, P. T. (1987). Control of linear systems using generalized sampled-data hold functions. IEEE Trans. Aut. Control, AC-32, 772-783.

Kaczorek, T. (1985). Pole assignment for linear discrete-time systems by periodic output feedbacks. Syst. Control Lett., 6, 267-269.

Kaczorek, T. (1986). Deadbeat control of linear discretetime systems by periodic output-feedback. IEEE Trans. Aut. Control, AC-31, 1153-1156.

Khargonekar, P. P., K. Poola and H. Tannenbaum (1985). Robust control of linear time-invariant plants using periodic compensation. IEEE Trans. Aut. Control, AC-30, 1088-1096.

Sirisena, H. R. (1985). Ripple-free deadbeat control of SISO discrete systems. IEEE Trans. Aut. Control, AC-30.

Tarn, T.-J., J. R. Zavgren and X. Zeng (1988). Stabilization of infinite dimensional systems with periodic feedback gains and sampled output. Automatica, 24, 95-99.

Urikura, S. and A. Nagata (1987). Ripple-free deadbeat control for sampled-data systems. IEEE Trans. Aut. Control, AC-32.

Wilson, D. A. (1970). Optimal solution of model reduction problems. Proc. IEEE, 17, 1161-1165.

Yang, C. and P. T. Kabamba (1988). Simultaneous design of sampled data control systems. In Proc. 1988 ACC, Atlanta, GA; to appear in IEEE Trans. Aut. Control.

Zavgren, J. R. (1983). Stabilisation of infinite-dimensional systems via periodic output feedback. Sc.D. Dissertation, Washington University, Saint Louis, MO.

Zavgren, J. R. and T.-J. Tarn (1984). Periodic output feedback stabilization of infinite-dimensional systems. Proc. 9th IFAC Triennial World Congress, pp. 1445-1449, Budapest, Hungary.

Zang, X. (1985). Sampled output stabilization of infinitedimensional systems. Sc.D. Dissertation, Washington University, Saint Louis, MO. 